\title{
A Fault Hiding Approach for the Sliding Mode Fault-tolerant Control of a Non-holonomic Mobile Robot
}

\author{
Alexandru Stancu ${ }^{1}$, Eduard Codres ${ }^{1}$ and Vicenç Puig ${ }^{2}$
}

\begin{abstract}
When mobile robots are intended to be used in hazardous environments or for long-time operations, it is needed to increase their robustness against faults. This could be achieved by means of the inclusion of Fault Tolerant Control (FTC) mechanisms. In this paper, a FTC based on fault hiding approach is proposed for a non-holonomic mobile robot. First, a Sliding Mode Controller (SMC) is designed to control the robost and to cope with modelling uncertainty. Later on, it is enhanced to take into account actuator faults leading to a fault hiding approach for the sliding mode faulttolerant control of the robot. Results using simulated fault scenarios are presented to illustrate the performance of the proposed approach.
\end{abstract}

\section{INTRODUCTION}

When mobile robots are intended to be used in hazardous environments or for long-time operations, it is needed to increase their robustness against failures. This could be achieved by means of the inclusion of Fault Tolerant Control (FTC) mechanisms in the robot. The objective of FTC is to maintain current performances close to desirable ones and preserve stability conditions in the presence of faults [6], [13]. The existing FTC design techniques mainly can be classified according if they follow the passive or the active approach [3]. The passive FTC techniques are control laws that take into account the fault as a system disturbance. Thus, within certain margins, the control law has inherent fault tolerant capabilities, allowing the system to cope with the fault presence [10]. On the other hand, the active FTC techniques compensate the faults either by selecting a precalculated control law or by synthesizing online a new control strategy. The adaptation of the control law is done by using some information about the fault, obtained from an on-line fault diagnosis system, so as to satisfy the control objectives with minimum performance degradation after the fault occurrence. Some examples of successful FTC strategies are multiple-model-based [2], learning-based [12] and adaptive backstepping-based [3], among other. Another, appealing strategy of active fault tolerant control is based on using the fault hiding approach that relies on adding some block between the controller and the system that hides the fault and avoids readjusting the controller. In case of faults of sensors and actuators, the virtual sensors and actuators are well known fault hiding blocks [6].

\footnotetext{
${ }^{1}$ Control Systems Department, University of Manchester, UK.

${ }^{2}$ Institut de Robòtica i Informàtica Industrial (CSIC-UPC). Carrer Llorens Artigas, 4-6, 08028 Barcelona.
}

In the formulation of any practical control problem using a model-based approach, there will always be a discrepancy between the actual system and its mathematical model used for the controller design. These discrepancies (or mismatches) arise from unknown external disturbances, system parameters, and parasitic/unmodeled dynamics. Designing control laws that provide the desired performance to the closed-loop system in the presence of these disturbances/uncertainties is a very challenging task for a control engineer. This has led to intense interest in the development of the so-called robust control methods which are supposed to solve this problem. One particular approach to robust controller design is the so-called sliding mode control (SMC) technique. SMC is a kind of robust control technique that can deal with large uncertainty with a discontinuous control strategy. The insensitivity and robustness of SMC make it suitable for handling the system under control with satisfactory performance in both normal and faulty operating conditions. These interesting features convert this control strategy in a very suitable approach for FTC. Recently, some advances in the design of FTC SMC have been summarised in the book of Alwi [4].

The application of the FTC to mobile robots is quite recent. Just few references can be found in the literature and summarised in [7]. The problem of FTC in robotics to increase their robustness against possible failures has been addressed in [11]. Four-wheeled omnidirectional mobile robots have the relevant characteristic that they can still operate with three wheels in case some malfunctioning in one wheel has been detected [9]. This makes them good setups for testing techniques that provide fault tolerance against actuator faults. Just recently, FDI and FTC approaches have been considered for multi-robot systems in [5], where a distributed controller-observer architecture was proposed. This allows each robot to estimate the global system state using local communication.

In this paper, a fault hiding approach for including FTC in a SMC is designed for a non-holonomic mobile robot. First, a SMC controller for trajectory tracking based on a kinematic model of the robot is designed to cope with modelling uncertainty. Later on, it is enhanced to take into account actuator faults leading to a FTC SMC by using a fault hiding approach. To the best of our knowledge, the approach proposed in this paper is novel and there is nothing similar in the literature 

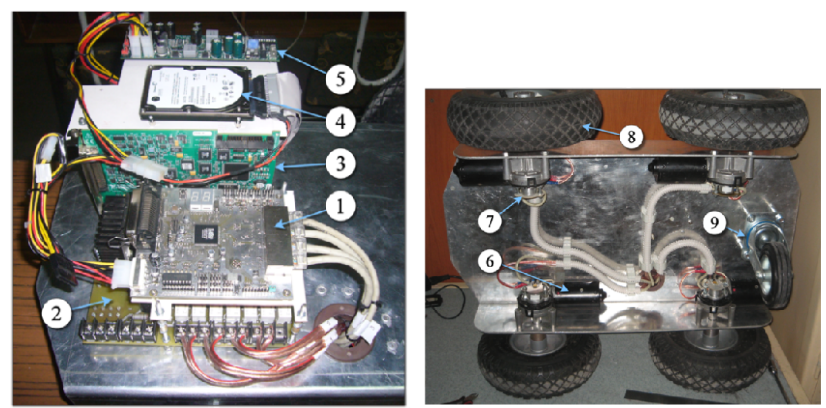

Fig. 1: Components of the four wheel non-holonomic robot. (1) Extension module. (2) DC motor drivers. (3) Acquisition card (NI). (4) HDD. (5) PC power supply. (6) Permanent magnet DC motors. (7) Incremental encoder 4096 pulse/revolution (Avago). (8) Driving wheel and (9) Castor wheel.

in mobile robotic applications. Results using simulated and real fault scenarios are presented to illustrate the performance of the proposed approach. The structure of the paper is the following: In Section II, the mobile robot is described. Section III presents the sliding mode controller for the mobile robot. Section IV extends the sliding mode controller to cope with faults and a new fault hiding approach for the sliding mode fault-tolerant control of a non-holonomic mobile robot is introduced. In Section $\mathrm{V}$, the proposed methods are illustrated in some fault scenarios. Conclusions and on-going work are outlined in Section VI.

\section{Mobile Robot Description}

\section{A. Robotic platform}

The mobile robotic platform considered in this paper is a four wheel non-holonomic robot built in the Autonomous Systems Lab at The University of Manchester, UK. The robot is presented in Fig. 1.

The purpose of this non-holonomic mobile robot is to provide a robotic platform to be used for benchmarking several fault monitoring (fault detection and isolation) and fault tolerant control strategies. It is well known that using off the shelf robotic platforms is not possible to access all the sub-components of the system. In order to be able to test the fault monitoring and fault tolerant control algorithms we often need to measure the currents in the DC motors, or to be able to send voltage as control signals (PWM) directly to the DC motors. To the best of our knowledge, the majority of off the shelf robotic platforms accept as control signals the angular velocities for each wheel since the voltage control is provided internally using a microcontroller. In this way, the user does not have access to the low level components of the robotic platform. To cope with this issue, the proposed robotic platform was designed in a modular way giving the possibility to the user to access every low level component. Thus, some components can be replaced with faulty ones in order to

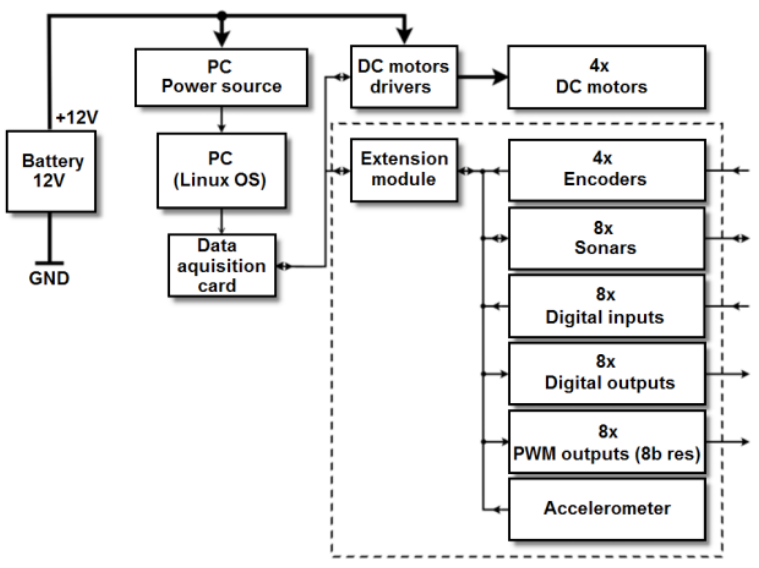

Fig. 2: Schematic representation of the main components of the robotic platform and the interdependencies among them.

study several fault monitoring and fault-tolerant control algorithms. Moreover, the robotic platform can be used in two different configurations. The first configuration is a mobile vehicle with four independent driving wheels. The second configuration is a mobile robot with two driving wheels and one caster wheel. The second configuration can be easy obtained by replacing two of the driving wheels with a caster wheel. In this way, two different configurations of mobile robots (with two and with four driving wheels) can be used as benchmark. In this paper, the configuration with two driving wheels and one caster wheel will be used to test the proposed fault tolerant control algorithm. Fig. 1 shows the main components of the proposed robotic platform. These components and the interdependencies are schematically illustrated in Fig. 2.

\section{B. Dynamic model for the robotic platform}

Although the kinematic approach is useful for control design, it is not accurate enough for the analysis of controllers dealing with uncertainty (robustness) and faults. Moreover, for an realistic simulation the kinematic model is not enough, being neccessary to develop a complete dynamic model. Using the Lagrange dynamical modeling approach, the dynamical model of a non-holonomic differential drive mobile robot can be described by the following equation:

$M(q) \ddot{q}+V(q, \dot{q}) \dot{q}+\tau_{d}=B(q) \tau-F_{R}(\dot{q})-\Lambda^{T}(q) \lambda$

$M(q)-n \times n$ inertia matrix;

$V(q, \dot{q})$ - Coriolis and centripetal matrix;

$F_{R}(\dot{q})$ - surface and rolling friction matrix;

$\tau_{d}-$ unknown disturbances and unstructured unmodeled dynamics;

$B(q)$ - input matrix;

$\tau$ - input torque vector;

$\Lambda^{T}$ - kinematic constraints matrix;

$\lambda$ - Lagrange multiplier; 


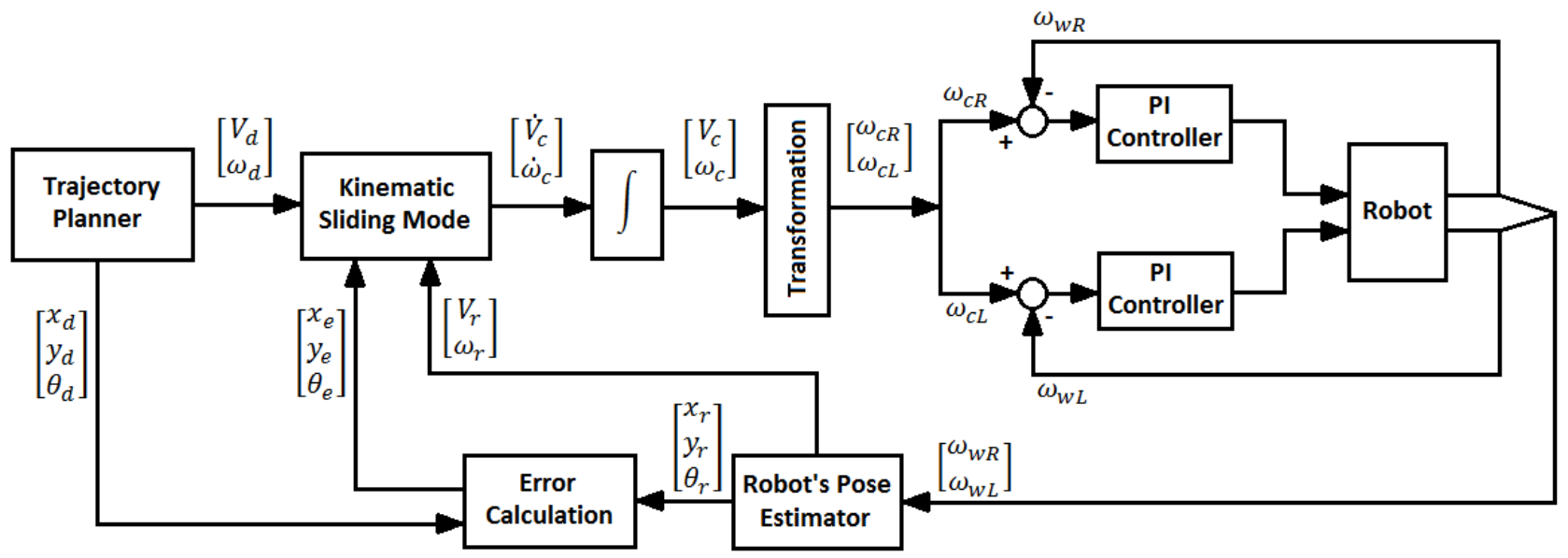

Fig. 3: Block diagram for SMC control based on kinematic model for trajectory tracking of the mobile robot

\section{SLIDING MOde CONTROL FOR TRAJECTORY TraCking BASEd on Kinematic Model}

\section{A. Introduction}

In this section, the design of SMC based on the kinematic model for trajectory-tracking for the mobile robot described in Section II is described. The proposed control strategy is illustrated in Fig. 3.

In order to develop a robust controller for wheeled mobile robot trajectory-tracking, we assume that the trajectory is a priori generated by a trajectory planner as it is proposed in [22]. The problem consists in designing a robust controller so that the robot will correctly track the desired trajectory under a large class of disturbances. The trajectory planner generates the desired positions, velocities and accelerations. The SMC is designed such that it will minimise the difference between the real positions, velocities, accelerations and the desired ones. The desired values are assigned to a virtual robot as it is shown in Fig. 4. The sliding mode controller based on kinematic model will provide the linear and angular velocities for the real robot in order to track the virtual one with as small errors as possible. The block "Robots Pose Estimator" will compute the robot positions and orientation $x_{r}, y_{r}, \theta_{r}$ (also known as robot pose or odometry) as well as the linear and the angular velocities of the robot $v_{r}, w_{r}$ [21].

The trajectory parameters are defined as follows:

- $x_{d}, y_{d}, \theta_{d}$ - desired pose;

- $v_{d}, w_{d}$ - desired linear and angular velocities;

- $\dot{v}_{d}, \dot{w}_{d}$ - desired linear and angular accelerations;

Then, the following motion model is considered as the model of the non-holonomic wheeled mobile robot:

$$
\left\{\begin{array}{c}
\dot{x}_{r}=v_{r} \cos \theta_{r} \\
\dot{y}_{r}=v_{r} \sin \theta_{r} \\
\dot{\theta}_{r}=w_{r}
\end{array}\right.
$$

where $\left[x_{r}, y_{r}, \theta_{r}\right]$ is the robot pose (the position of the middle of driving axis and the orientation), $v_{r}$ is the forward velocity and $w_{r}$ is the angular velocity of the robot.

The tracking error vector $\left[x_{e}, y_{e}, \theta_{e}\right]^{T}$ is defined as a transformation in the robot frame:

$$
\left[\begin{array}{l}
x_{e} \\
y_{e} \\
\theta_{e}
\end{array}\right]=\left[\begin{array}{ccc}
\cos \theta_{d} & \sin \theta_{d} & 0 \\
-\sin \theta_{d} & \cos \theta_{d} & 0 \\
0 & 0 & 1
\end{array}\right]\left[\begin{array}{l}
x_{r}-x_{d} \\
y_{r}-y_{d} \\
\theta_{r}-\theta_{d}
\end{array}\right]
$$

The corresponding error derivatives are:

$$
\left\{\begin{array}{c}
\dot{x}_{e}=-v_{d}+v_{r} \cos \theta_{e}+y_{e} w_{d} \\
\dot{y}_{e}=v_{r} \sin \theta_{e}-x_{e} w_{d} \\
\dot{\theta}_{e}=w_{r}-w_{d}
\end{array}\right.
$$

Slotine proposes in [23] the following type of switching manifold for trajectory tracking:

$$
s=\dot{\tilde{x}}+k \tilde{x}
$$

where $\tilde{x}=x-x_{\text {desired }}$.

In the ideal case, $s=0$ and the unique solution of this differential equation is $\tilde{x}=0$. An important aspect of the invariant set $s$ is that once on it, the system trajectories are defined by the equation of the set itself, namely $\dot{\tilde{x}}+k \tilde{x}=0$. Hence, the surface $s$ is both a place and a dynamic.

\section{B. The Proposed SMC}

In this paper, a new design strategy of the sliding surfaces is proposed. Since in the trajectory tracking problem we have three variables $\left(x_{e}, y_{e}, \theta_{e}\right)$ and only two control variables (the linear and angular velocity of the mobile robot $v_{c}$ and $w_{c}$ respectively), we propose to couple the lateral error $y_{e}$ and the angular error $\theta_{e}$ in one sliding surface $s_{2}$ as follows

$$
\begin{aligned}
& s_{1}=\dot{x}_{e}+k_{1} x_{e} \\
& s_{2}=\dot{\theta}_{e}+k_{2} \theta_{e}+k_{0} y_{e}
\end{aligned}
$$




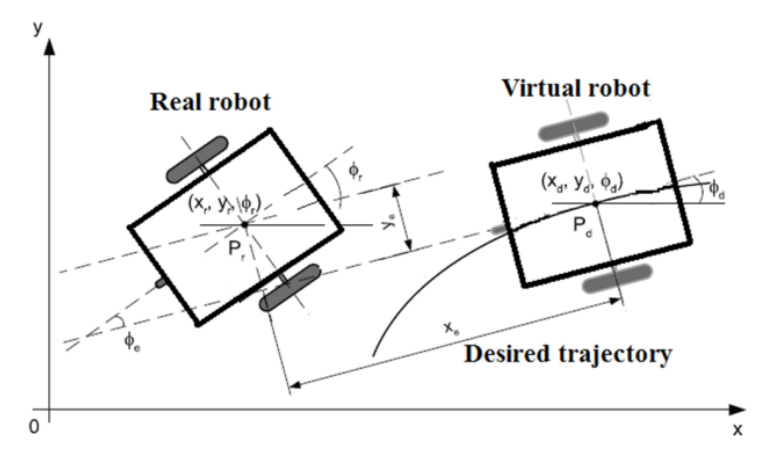

Fig. 4: The errors between the real robot and the virtual one

where $k_{0}, k_{1}, k_{2}$ are the parameters of the sliding surfaces. The values for these parameters will be chosen such that the surfaces $s_{1}$ and $s_{2}$ are stable, i.e., the tracking errors converge to zero when $s_{1}=0$ and $s_{2}=0$ (see Proposition 1).

Proposition 1: : The sliding surfaces $\left(s_{1}\right.$ and $\left.s_{2}\right)$ in (6) are asymptotically stable if

$$
k_{0}>\frac{k_{2}\left|\theta_{e}\right|}{\left|y_{e}\right|}, k_{1}>0, \text { and } k_{2}>0
$$

Then, the tracking errors $x_{e}, y_{e}$ and $\theta_{e}$ converge asymptotically to zero.

Proof. Follows directly applying the Lyapunov approach.

Proposition 1 proves that once the errors are on the sliding surfaces they will remain there forever. This working regime is also known as sliding phase. If the errors are not on the sliding surfaces, a control law is needed in order to bring the errors back to the sliding manifolds. This working regime is also known as the reaching phase.

Proposition 2: If the system is in the reaching phase, the control law given by [8],

$$
\dot{s}=-Q s-P \operatorname{sgn}(s)
$$

where $s=\left[\begin{array}{ll}s_{1} & s_{2}\end{array}\right]^{T}$, moves the system towards the sliding phase in a stable way.

The time derivatives of $s_{1}$ and $s_{2}$ are

$$
\begin{aligned}
& \dot{s}_{1}=\ddot{x}_{e}+k_{1} \dot{x}_{e}=\dot{v}_{r} \cos \theta_{e}+\dot{v}_{r} \sin \theta_{e}+ \\
& \dot{y}_{e} w_{d}+y_{e} \dot{w}_{d}-\dot{v}_{d}+k_{1} \dot{x}_{e} \\
& \dot{s}_{2}=\ddot{\theta}_{e}+k_{2} \dot{\theta}_{e}+k_{0} \dot{y}_{e}=\dot{w}_{r}-\dot{w}_{d}+k_{2} \dot{\theta}_{e}+k_{0} \dot{y}_{e}
\end{aligned}
$$

From (7), (9), (14), (16) and (17), and after some basic mathematical manipulation, we get the derivatives of control signals, i.e., the derivative of the linear and angular velocity of the mobile robot, $\dot{v}_{c}$ and $\dot{w}_{c}$ respectively.

$$
\begin{aligned}
& \dot{v}_{c}=\left(-Q_{1} s_{1}-P_{1} \operatorname{sgn}\left(s_{1}\right)-v_{r} \dot{\theta}_{e} \sin \theta_{e}-\dot{y}_{e} w_{d}-\right. \\
& \left.y_{e} \dot{w}_{d}+\dot{v}_{d}-k_{1} \dot{x}_{e}\right) \frac{1}{\cos \theta_{e}} \\
& \dot{w}_{c}=-Q_{2} s_{2}-P_{2} \operatorname{sgn}\left(s_{2}\right)+\dot{w}_{d}-k_{2} \dot{\theta}_{e}-k_{0} \dot{y}_{e}
\end{aligned}
$$

where $k_{0}>\frac{k_{2}\left|\theta_{e}\right|}{\left|y_{e}\right|}, k_{1}>0$, and $k_{2}>0$ (see Proposition 1 ), and $P_{1}>0, P_{2}>0, Q_{1}>0$ and $Q_{2}>0$ (see Proposition 2).

After integration, we get the control linear $\left(v_{c}\right)$ and angular $\left(w_{c}\right)$ velocities of the mobile robot. These velocities can be transformed in angular velocities for left and right wheel $w_{c L}$ and $w_{c R}$, respectively. The angular velocities of the wheels will be applied to the robots wheels by means of an inner control loop based on a PI controller as it is shown in Fig. 3. Considering that the wheels have a radius $R$ with a distance of separation $L$, and assuming a perfectly symmetric body frame, the resultant forward velocity can be obtained as an average of the two forward wheel velocities given by $R\left(\frac{w_{c R}+w_{c L}}{2}\right)$. The steering velocity may also be reasoned as proportional to the difference between wheel velocities but inversely proportional to distance between the wheels, i.e., $R\left(\frac{w_{c R}-w_{c L}}{2}\right)$. Thus

$$
\begin{aligned}
& w_{c R}=\frac{v_{c}+L w_{c}}{R_{R}} \\
& w_{c L}=\frac{v_{c}-L w_{c}}{R_{L}}
\end{aligned}
$$

where $w_{c R}$ and $w_{c L}$ are the angular velocities applied to the right and left wheels, respectively.

\section{InCLUding FAUlt Hiding MECHANISM}

Previous SMC control scheme for the robot presented in Section III can be adapted to handle faults. Here, for illustrative purposes and without reducing the generality of the proposed fault-tolerant control method, the case of fault affecting the wheel radius (flat tire) is considered. There are two ways of including the faults in the SMC control scheme: the SMC could be readjusted on-line, or alternatively, a fault hiding approach can be used without need of retuning the controller. Here, the second option is the one selected. In Fig. 5, the SMC scheme proposed in Fig. 3 is modified by including a block which contains the fault hiding mechanism for each of the active wheels of the mobile robot. The role of this block is to hide the effect of fault to the SMC avoiding to retune it.

In the following proposition, we will show that the fault hiding mechanism is of the form $\frac{\Delta R}{R_{n o m}} \dot{\nu}_{c}$ and $\frac{\Delta R}{R_{\text {nom }}} \dot{\omega}_{c}$, where $R_{n o m}$ is the nominal radius of the wheel and $\Delta R$ is the maximum range of radius variation. Moreover, it will be shown that the sliding mode controller based on kinematic model together with fault hiding block is stable. 


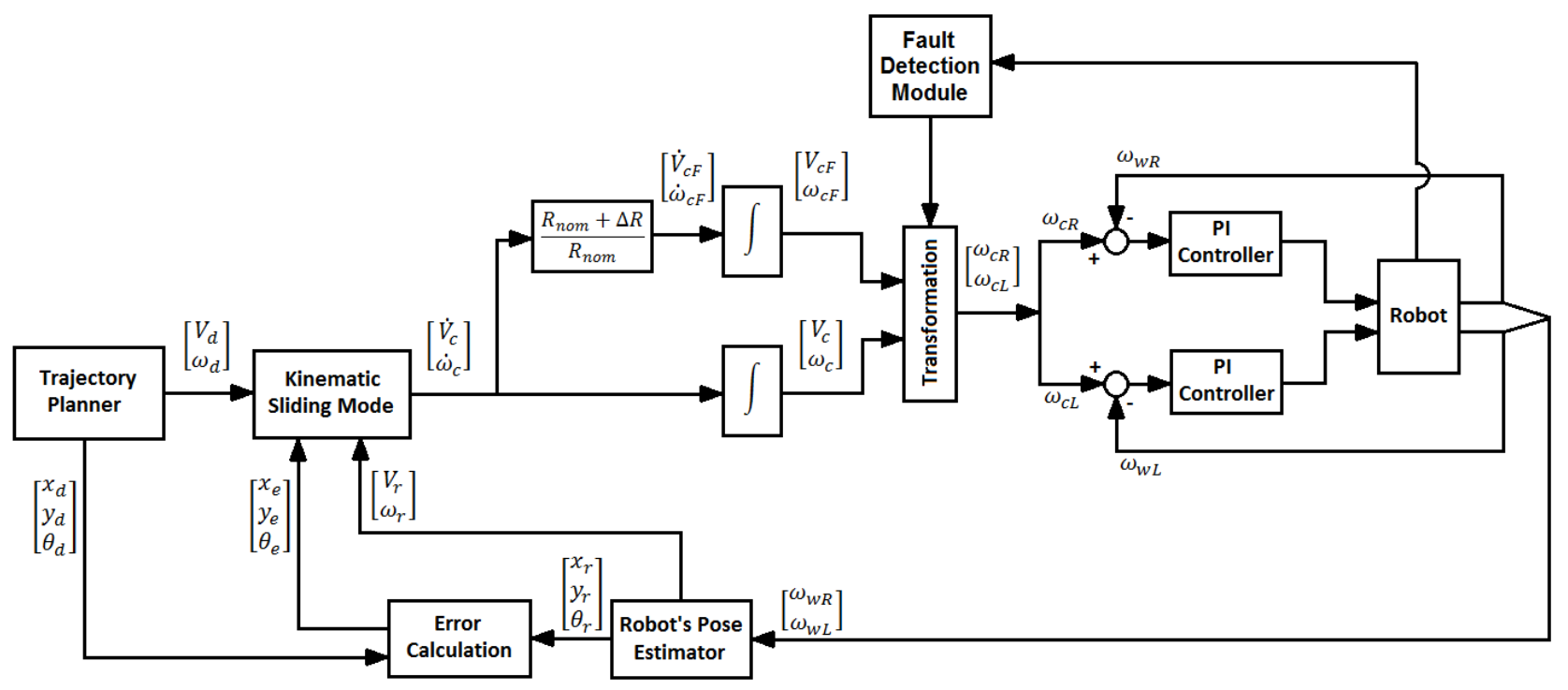

Fig. 5: Fault Hiding for the Sliding Mode Fault-tolerant Control of a Non-holonomic Mobile Robot for fault in the actuators (flat tire).

Proposition 3: Given a robot with two active wheels, the following fault accommodation mechanism

$$
\begin{aligned}
& \dot{\nu}_{\text {fault }}=\frac{R_{\text {nom }}+\Delta R}{R_{\text {nom }}} \dot{\nu}_{c} \\
& \dot{\omega}_{\text {fault }}=\frac{R_{\text {nom }}+\Delta R}{R_{\text {nom }}} \dot{\omega}_{c}
\end{aligned}
$$

is stable and robust against faults in the mobile robot wheels.

Proof. Follows directly applying the Lyapunov approach.

Remark 3: If $\Delta R=0$, the derivatives of linear and angular velocities of the robot obtained using SMC based on the simplified dynamic model becomes identical with the ones obtained using SMC based on the kinematic model, i.e., $\dot{\nu}_{\text {fault }}=\dot{\nu}_{c}$ and $\dot{\omega}_{\text {fault }}=\dot{\omega}_{c}$.

Considering the faults which can occur in the wheels of the mobile robot (flat tire) we can distinguish the following three cases.

Case 1. Both wheels are faulty:

$$
\begin{aligned}
& R_{R f}<R_{n o m} \text { and } R_{R f}<\Delta R \\
& R_{L f}<R_{n o m} \text { and } R_{L f}<\Delta R
\end{aligned}
$$

The angular velocities for each wheel will be:

$$
\begin{aligned}
& \omega_{c R f}=\frac{\nu_{\text {fault }}+L \omega_{\text {fault }}}{R_{\text {nom }}} \\
& \omega_{c L f}=\frac{\nu_{\text {fault }}-L \omega_{\text {fault }}}{R_{\text {nom }}}
\end{aligned}
$$

where $R_{R f}, R_{L f}$ are the radius of the right and left faulty wheels, respectively. $\omega_{c R f}$ and $\omega_{c L f}$ are the angular velocities of the right and left faulty wheels, respectively.
Case 2. Left wheel is faulty, right wheel is non faulty:

$$
\begin{aligned}
R_{R} & =R_{n o m} \\
R_{L f} & <R_{n o m} \text { and } R_{L f}<\Delta R
\end{aligned}
$$

The angular velocities for each wheel will be:

$$
\begin{aligned}
\omega_{c R f} & =\frac{\nu_{c}+L \omega_{c}}{R_{R}} \\
\omega_{c L f} & =\frac{\nu_{\text {fault }}-L \omega_{\text {fault }}}{R_{\text {nom }}}
\end{aligned}
$$

Case 3. Left wheel is non faulty, right wheel is faulty:

$$
\begin{aligned}
R_{L} & =R_{n o m} \\
R_{R f} & <R_{n o m} \text { and } R_{R f}<\Delta R
\end{aligned}
$$

The angular velocities for each wheel will be:

$$
\begin{gathered}
\omega_{c L}=\frac{\nu_{c}-L \omega_{c}}{R_{L}} \\
\omega_{c R f}=\frac{\nu_{\text {fault }}+L \omega_{\text {fault }}}{R_{\text {nom }}} \\
\text { V. RESULTS }
\end{gathered}
$$

\section{A. Testing set-up}

In this section, using a validated mobile robot model (presented in Section II.B), simulated results of the proposed fault-tolerant control method are presented in fault free scenarios and faulty scenarios. The simulation is used in order to be able to "inject" faults in the wheel at any time instant. Using the model in Section II.B as virtual reality, it is possible to analyse the proposed fault-tolerant control strategy for faults which appear at any time instant. This is not possible for the real robot 
in which case the fault is considered to be present before we start the experiment.

We will consider a circular trajectory for all test cases. The circular reference trajectory was generated a priori using an algorithm based on quintic equations as it was proposed by Solea in [22]. The dynamic model was simulated in Matlab ${ }^{\circledR}$ using the following physical parameters identified from the real mobile robot:

\section{Robot parameters:}

Right wheel radius: $R_{R}=0.1145 \mathrm{~m}$;

Left wheel radius: $R_{R}=0.1145 \mathrm{~m}$;

Half of wheel base: $L=0.245 \mathrm{~m}$;

Distance between center of mass and centre of rotation: $d=0.1 \mathrm{~m}$;

Robot body mass: $M_{B}=35 \mathrm{Kg}$;

Wheel mass: $W_{w}=0.35 \mathrm{Kg}$;

Robot body moment of inertia: $I_{B}=1.5 \mathrm{Kg} \cdot \mathrm{m}^{2}$;

Wheel moment of inertia around robot axis of rotation: $I_{M}=0.0012 \mathrm{Kg} \cdot \mathrm{m}^{2}$;

Wheel moment of inertia around actuator axis of rotation: $I_{W}=0.0012 \mathrm{Kg} \cdot \mathrm{m}^{2}$;

Static friction for right wheel: $C_{S R}=2.35$;

Static friction for left wheel: $C_{S L}=2.73$;

Viscous friction for right wheel: $C_{V R}=0.17$;

Viscous friction for left wheel: $C_{V L}=0.25$;

\section{DC motor parameters:}

DC motor torque constant: $K_{t e}=0.03$;

The resistance of the armature windings: $R_{e}=1.8 \Omega$;

The DC motor inductance: $L_{e}=0.08 \mathrm{H}$;

Gearbox multiplication: $N_{G}=40$;

\section{Input voltage:}

The voltage applied in open loop to the right DC motor: $V_{R}=9.5 \mathrm{~V}$;

The voltage applied in open loop to the left DC motor: $V_{L}=8.5 \mathrm{~V}$;

\section{B. Simulation results of SMC based on kinematic model in non-faulty case}

Design parameters of (6) and (8) were chosen in accordance with Proposition 1 and Proposition 2 in order to guarantee the errors convergence by keeping the surfaces $s_{1}$ and $s_{2}$ to zero (sliding phase) and to guarantee the convergence of the control law (reaching phase). Moreover, in order to reduce the chattering phenomenon the signum functions in the control law (14) were replaced by saturation functions with the threshold \pm 0.5 . Fig. 6a-6g show the results for trajectory-tracking using SMC based on kinematic model in fault free scenario. It can be seen that linear and angular velocities of the mobile robot (Fig. 6c and Fig. 6d) follow the references generated a priori by the Trajectory Planner. The simulated mobile robot is able to

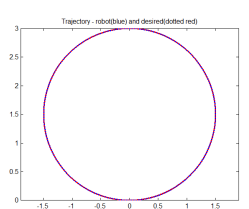

(a)

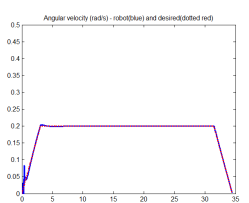

(d)

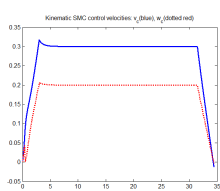

(g)

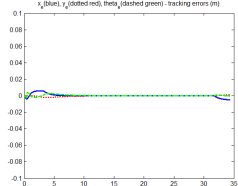

(b)

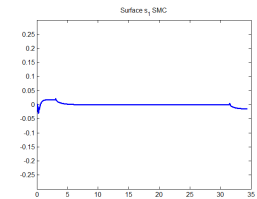

(e)

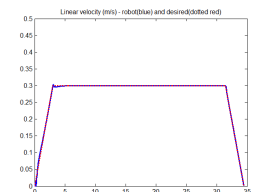

(c)

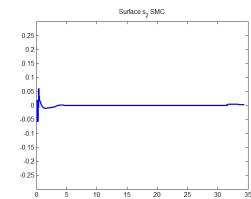

(f)
Fig. 6: Simulation results of trajectory tracking using a SMC based on a kinematic model in a non-faulty situation

track with small errors the virtual robot (see Fig. 6a). It is also shown that the sliding surfaces $s_{1}$ and $s_{2}$ are kept to zero (Fig. 6e and Fig. 6f). Consequently (see Proposition 1 ), the tracking errors converge to zero Fig. 6b, as well. In Fig. $6 \mathrm{~h}$ the angular velocities for both wheels right and left are shown. These angular velocities are maintained by means of a simple PI controller as it was shown in Fig. 3.

\section{Simulation results of SMC based on kinematic model in faulty case}

The fault is introduced in the left wheel (flat tire of the left wheel). The radius of the left wheel is changed from $R_{L}=R_{n o m}=0.1145$ meters to $R_{L f}=0.0745$ meters and it is "injected" to the robot model at $t=16$ seconds and will be faulty for the rest of the simulation. In this case, we do not consider the fault hiding block and the trajectory-tracking is based on the control strategy proposed in Section III (see Fig. 3), i.e., SMC based on kinematic model of the mobile robot.

In Fig. 7a one can observe the tracking errors in the moment when the fault occurs ( $t=16$ seconds). After a finite time the control law [8] manages to make both sliding surfaces zero (Fig. 7d and Fig. 7e) and consequently the tracking errors (Fig. 7a). In Fig. $7 \mathrm{~g}$ it can be seen that the angular velocity of the faulty wheel (dotted red line) increases as result of a big control effort needed to overcome the fault effect (the control velocities observed in Fig. 7f). In Fig. $7 \mathrm{~b}$ and $7 \mathrm{c}$ it can be seen the real linear and angular velocities of the robot and their variations when the fault occurs. The SMC based on kinematic model is robust even in presence 


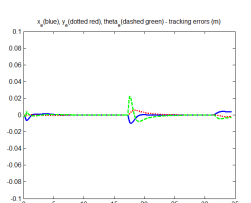

(a)

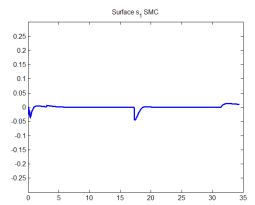

(d)

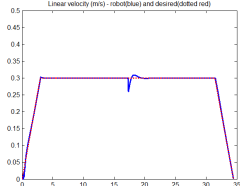

(b)

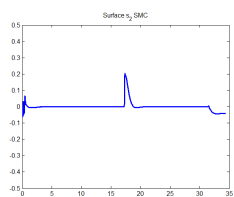

(e)

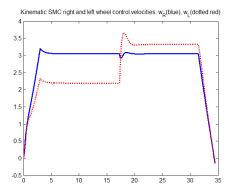

(g)

Fig. 7: Simulation results of trajectory tracking using a SMC based on a kinematic model in a faulty situation without fault hiding block

of the fault in the left wheel thanks to the robustness properties of the SMC strategy. Since the fault can be of any magnitude, we cannot guarantee the robustness against any possible faults. In order to deal with this situation we have, somehow, to take into account during the controller design phase, the fault magnitude and to prove the robustness of the fault tolerant controller against a maximum possible fault magnitude $(\Delta R)$. In this way, we can guarantee the robustness of the fault-tolerant controller (SMC plus fault hiding block) for all possible faults with magnitudes less or equal than the maximum one considered.

Now, we will apply the fault hiding strategy proposed in Section IV, see Fig. 5. Here, we consider the maximum range of radius variation to be $\Delta R=0.06$ meters. From Fig. $8 \mathrm{a}-8 \mathrm{f}$, it can be seen that because of the fault hiding approach considered, the SMC based on kinematic model is almost insensitive to the fault in the left wheel, occurred at $t=16$ seconds, comparing with the sensitivity in the case of SMC without the fault hiding block as it was shown in Fig. 7a-7f. In Fig. 8f, it can be seen that after just few iterations (the time need for the fault hiding block to become active), the control signals (linear and angular velocities of the mobile robot) have the same values as in the case of fault free situation (Section V.B), Fig. 6g. The responsible of the change in the angular velocity for the left wheel (dotted red in Fig. 8g) after the fault occurrence is the fault hiding block and not the SMC which remains insensitive for the rest of the simulation (see Fig. 8f).

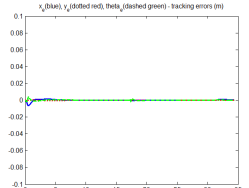

(a)

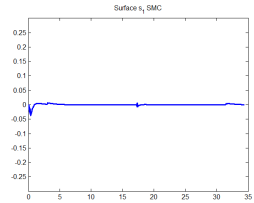

(d)

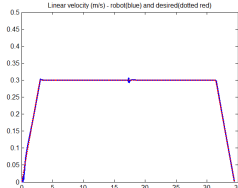

(b)

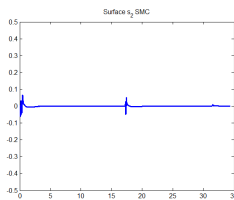

(e)

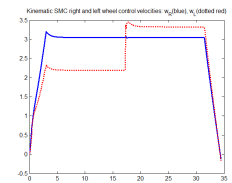

(g)

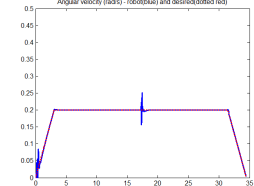

(c)

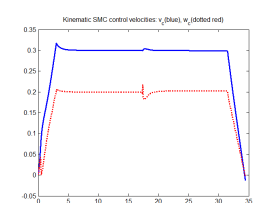

(f)
Fig. 8: Simulation results of trajectory tracking using a SMC based on a kinematic model in a faulty situation with the fault hiding block

\section{CONCLUSIONS}

In this paper, a fault hiding approach to include FTC capabilities to SMC based on kinematic model for a mobile robot was proposed. It has been proved that the SMC based on kinematic model with the fault hiding block can cope with faults in the actuators for a mobile robot. Moreover, using a sliding mode controller based on a reduced dynamic model for the mobile robot it was possible to prove the stability of the sliding mode based on kinematic model working together with the fault hiding block in case of fault occurrence. In this way, we propose a simple fault hiding block to be added to the SMC based on kinematic model (used in the majority papers and applications of trajectory-tracking for mobile robots) in order to cope with faults in the actuators (flat tire). Results using simulated fault scenarios were presented to illustrate the performance of the proposed approach.

As future work we will take into account other possible faults for mobile robots, as for instance the variance of the robots mass and consequently the moment of inertia. For this type of fault we will apply the same strategy as the one proposed in this paper.

\section{ACKNOWLEDGMENT}

This work has been partially funded by the Spanish Government (MINECO) through the project CICYT ECOCIS (ref. DPI2013-48243-C2-1-R), by MINECO and FEDER through the project CICYT HARCRICS (ref. DPI201458104-R). 


\section{REFERENCES}

[1] H. Alwi, C. Ed and P. W. Daly, A Guide to $B T_{E} X$, 3rd ed. Harlow, England: Addison-Wesley, 1999.

[2] G. G. Yen and L.-W. Ho, Online multiple-model-based fault diagnosis and accommodation, IEEE Trans. Ind. Electron., vol. 50, no. 2, pp. 296 312, Apr. 2003.

[3] X. Zhang, T. Parisini, and M. M. Polycarpou, Adaptive fault-tolerant control of nonlinear uncertain systems: An information-based diagnostic approach, IEEE Trans. Autom. Control, vol. 49, no. 8, pp. 12591274, Aug. 2004.s

[4] H. Alwi, C. Edwards and Pin Tan, Fault Detection and Fault-Tolerant Control Using Sliding Modes, Springer-Verlag, 2011.

[5] F. Arrichiello, A. Marino and F. Pierri, A Decentralized Fault Tolerant Control Strategy for Multi-Robot Systems, Proceedings of the 19th IFAC World Congress, Cape Twon, South Africa, 2014

[6] M. Blanke, M. Kinnaert, J. Lunze, and M. Staroswiecki, Diagnosis and Fault-Tolerant Control Springer-Verlag, Berlin Heidelberg, 2006.

[7] D. Crestani and K.Godary-Dejean, Fault Tolerance in Control Architectures for Mobile Robots: Fantasy or Reality?. CAR 2012 : 7th National Conference on Control Architectures of Robots, May 2012, Nancy, France. pp.10.

[8] W. Gao, J. C. Hung, Variable structure control of nonlinear systems: A new approach IEEE Transactions on Industrial Electronics, no. 40, pp. $4555,1993$.

[9] A. Gloye, F. Wiesel, O. Tenchio, M. Simon and R. Rojas, Robot heal thyself: Precise and fault-tolerant control of imprecise and malfunctioning robots, in Proc. RoboCup Int. Symp., pp. 112, 2005.

[10] J. Jiang and X. Yu, Fault-tolerant control systems: a comparative study between active and passive approaches, Annual Reviews in Control, vol. 36, no. 1, pp. 6072, 2012.

[11] M. J. Jung and J.H. Kim, Development of a fault-tolerant omnidirectional wheeled mobile robot using nonholonomic constraints, Int J. Robot. Res., vol. 21, no. 56, pp. 527539, May 2002.

[12] M.M. Polycarpou, Fault accommodation of a class of multivariable nonlinear dynamical systems using a learning approach, IEEE Transactions on Automatic Control, vol. 46, no. 5, pp. 736742, 2001.

[13] H. Noura, D. Theilliol, J. C. Ponsart, and A. Chamseddine, Fault-tolerant control systems: Design and Practical Applications, Springer-Verlag London Limited, 2009.

[14] V. I. Utkin, Variable structure systems with sliding mode, IEEE Transactions on Automatic Control, pp. 212-222, 1977.

[15] B. Drazenovic, The Invariance Conditions in Variable Structure Control, Automatica, pp. 287-295, 1969.

[16] L.E. Aguilar, T. Hamel and P. Soueres, Robust Path Following Control for Wheeled Robots via Sliding Mode Techniques, Intelligent Robots and Systems - IROS 97, 3, 13891395, 1997.

[17] D. Chwa, Sliding-Mode Tracking Control of Nonholonomic Wheeled Mobile Robots in Polar Coordinates, IEEE Transactions on Control Systems Technology, 12, (637644), 2004.

[18] R. Fierro and F.L. Lewis, Control of a nonholonomic mobile robot: backstepping kinematics into dynamics, 34th Conference on Decision and Control, New Orleans, 3805-3810, 1995.

[19] J.M. Yang and J.H. Kim, Sliding Mode Control for Trajectory Tracking of Nonholonomic Wheeled Mobile Robots, IEEE Transactions on Robotics and Automation, 15, (578 587), 1999.

[20] G.F. Yuan, Tracking control of a mobile robot using neural dynamics based approaches, Masters Abstracts International, 39, 1437, 2001.

[21] A. Stancu, E. Codres, and V. Puig. Mobile robotic platform for testing fault monitoring and fault tolerant algorithms, Internal report, University of Manchester, 2014.

[22] R. Solea, Sliding Mode Control Applied in Trajectory-Tracking of WMRs and Autonomous Vehicles, PhD thesis, 2009.

[23] J.J.E. Slotine and W. Li, Applied Nonlinear Control, Prentice-Hall Inter. Ed., 1991. 